\title{
Improving Connectivity of Cognitive Radio VANETs
}

\author{
Krishan Kumar ${ }^{\# 1}$, Mani Shekhar ${ }^{\# 2}$ \\ ${ }^{\#}$ Electronics and Communication Engineering Department, \\ National Institute of Technology, Hamirpur., India \\ ${ }^{1}$ krishan_rathod@nith.ac.in \\ 2mi_sr87@yahoo.com
}

\begin{abstract}
The current trends in vehicular adhoc networks are bringing a variety of changes to the existing one. The primary focuses in the area of vehicular adhoc networks are connectivity and file distribution which is broadcast by roadside units to the onboard units during handoffs. The connectivity of vehicular adhoc network can be improved by the use of cognitive radio networks with interference management approach. Then the networks are known as cognitive radio vehicular adhoc networks. The interference management approach can be done cooperatively where each individual node assign weights on the channel and short their channels to reduce the interference. But this type of interference approach is unnecessary in the highway as the spectrum remains low and clean. The other possible disadvantage is that the node shares its information with other nodes in the networks. Its solution is to use multiple antennas to improve the connectivity in cognitive radio vehicular adhoc networks which improves the data rates in highways. This paper proposes the approach to use of multiple antennas to improve the connectivity of cognitive radio vehicular adhoc networks during handoffs. The modification is done in available non-cooperative approach and coalitional graph game based approach. The simulation results show that the proposed approach increases the data rate compared to available approaches, reducing the interference and improving the connectivity in cognitive radio vehicular adhoc networks.
\end{abstract}

Keyword-Cognitive radio, CR VANETs, connectivity, antenna, non-cooperation, cooperation and handoffs.

\section{INTRODUCTION}

The current trends in wireless networking have raised the demand of extra spectrum bands. But the current wireless networks are bound with fixed spectrum allocation policy which is being regulated by the government agencies. The current fixed spectrum allocation policies used by the government agencies are unable to accommodate the growing demand of the wireless networks [1], [2]. As per Federal Communication Commission (FCC), up to $85 \%$ of the allocated spectrum is underutilized due to allocation of fixed spectrum bands. This produces the large gap between current increased demand of extra spectrum bands and fixed spectrum allocation policy. To overcome this gap, FCC has suggested to use the underutilized spectrums and spectrum portions which are not in use called vacant spectrum bands or white spaces [3], [4], [5], [6]. This generates the new area of research and development of Cognitive Radio Networks (CRNs) technology which is a key enabling technology that uses underutilized spectrum bands [7], [8].

The current trends in vehicular adhoc networks (VANETs) are bringing a variety of changes to the existing [9], [10], [11]. The VANETs are envisioned to provide connectivity to vehicle to vehicle (V2V) and vehicle to roadside (V2R). The data are handoffs between OBUs and between OBUs and RSUs. The connectivity of VANET scan be improved by the use of cognitive radio networks, called cognitive radio vehicular adhoc networks (CR VANETs) [12]. By using CR, it is possible to use other unused available channels with in better condition with increased data rate. Hence, the main advantage CR VANETs is that it does not require additional bandwidth and provides enhanced data rate. The main issue with CR VANET is connectivity to communicate data among nodes [13]. [13] Discussed CR technologies for VANETs aimed at improving its communication efficiency via dynamic spectrum access, adaptive software defined radio and cooperation etc. [14] study the analytical approach in wireless adhoc networks which says that complete cooperation and non-cooperation are not a proper approach to communicate data among nodes. [15] Discussed coalitional graph games for popular content distribution in CR VANETs. Its aim is to enhance the connectivity of CR VANETs when data are been transmitted during handoffs with the interference management approach. The interference management approach is done via non-cooperation approach and cooperation approach. The cooperation approach is done by coalitional graph game theory model in which OBUs self-organize into coalitions to coordinate their V2V and V2R communications in CR VANETs. But this type of interference approach is unnecessary in the highway as the spectrum remains low and clean. The other possible disadvantage is that the node shares its information with other nodes in the networks.

Its solution is to use multiple antennas to improve the connectivity in CR VANETs which improves the data rates which is the main contribution of this paper. This paper proposes the approach to use of multiple antennas 
to improve the connectivity of CR VANETs during handoffs. The modifications are done in non-cooperative approach and coalitional graph game based approach available in [15]. The simulation results show that the proposed approach increases the data rate compared to available approaches, reducing the interference and improving the connectivity in CR VANETs.

The rest of paper is organized as follows. Section II provides system model and available approaches. Section III provides the proposed approach: multiple antennas based modified approach. In section IV, numerical results are presented and section $\mathrm{V}$ provides the conclusion of this paper and scope of future work.

\section{SySTEM Model With AVAilable APPROACHES}

Let single omnidirectional antenna is used in OBUs and RSUs of CR VANET. The distance between RSUi and OBUi is assumed to be $\mathrm{d}_{\mathrm{i}}$.In case of V2R communication, the gain of channel between RSUi and OBUiis given by [15]

$$
\mathrm{h}_{\mathrm{i}}=\alpha \mathrm{d}_{\mathrm{i}}^{-2}
$$

where $\alpha$ is complex Gaussian random variable with unit variance and zero mean.

The corresponding channel capacity between RSUi and OBUi is given by [15]

$$
c_{i}=W \log _{2}\left(1+\beta\left|h_{i}\right|^{2}\right)
$$

whereW is the bandwidth for the channel between RSU and OBU.

$\beta$ is a scale factor which is the transmit power of RSU.

The pure broadcasting schemes do not allow V2V communication in VANETs.

During V2V communication, the channel gain $\mathrm{h}_{\mathrm{i}, \mathrm{j}}^{\mathrm{k}}, \mathrm{i} \neq \mathrm{j}$ between OBUs in the $\mathrm{k}^{\text {th }}$ unauthorized channel is given by as above if line of sight link is present between OBUs. The channel capacity is given by [15]

$$
\mathrm{c}_{\mathrm{i}, \mathrm{j}}^{\mathrm{k}}=\hat{\mathrm{W}}_{\mathrm{k}} \log _{2}\left(1+\beta_{\mathrm{i}}\left|\mathrm{h}_{\mathrm{i}, \mathrm{k}}^{\mathrm{k}}\right|^{2}\right), \mathrm{k}=1,2, \ldots \ldots \ldots, \mathrm{K}
$$

where $\hat{W}_{\mathrm{k}}$ is the bandwidth for the channel $\mathrm{k}$ between OBUs.

$\beta_{\mathrm{i}}$ is a scale factor which is the transmit power of OBUi.

A. Non-Cooperative Approach: In non-cooperative approach, the nodes do not cooperate with each other when the packets are handoff between different nodes. The packets are requested at the beginning of the time slot having the time period of each slot is $\mathrm{T}$.

The remaining transmit time left is $\left(T-k_{j} \tau\right)$ where $k_{j}$ is sensed channels from the total channels and $\tau$ is constant time. The transmission rate is given by [15]

$$
\mathrm{R}_{\mathrm{j}, \mathrm{i}}^{\mathrm{k}}=\left(\frac{\mathrm{T}-\mathrm{K}_{\mathrm{j}} \tau}{\mathrm{T}}\right) c_{j, i}^{k}, \mathrm{k} \in \mathrm{k}_{\mathrm{j}}
$$

The OBU selects the channel with largest transmission rate and the channel capacity which is a function of $\mathrm{f}\left(\mathrm{k}_{\mathrm{j}}\right)$. Let $\mathrm{f}\left(\mathrm{k}_{\mathrm{j}}\right)=\operatorname{Aln}\left(\mathrm{k}_{\mathrm{j}}+1\right)$ and $\mathrm{T}=\mathrm{k} \tau$. Now, the transmission rate can be written again as follows [15]

$$
\left(k_{j}+1\right) \ln \left(k_{j}+1\right)+\mathrm{k}_{j}=\mathrm{k}
$$

B. Coalitional Graph Game Based Approach: This is game theory approach in which OBUs self-organize into coalitions to coordinate V2V and V2R communication when the packets are handoff. Here OBUs connects the other one to maximize its own utility. The utility function is graph based function explained in [15].

In this paper, the probability of false alarm, $P_{f}$ and the probability of miss detection, $P_{m}$ are assumed to be fixed. The work does not try to show the impact of these parameters. Calculation of these parameters under various environmental conditions can be the extension of this work.

Let $P_{0}$ is the probability that there is no primary user in the channels during V2V communication. The total number of available channels are $P_{0 k}$. OBU $i \in \mathrm{N}_{\mathrm{i}}$ has occupied one of them. Then, the probability that OBU $\mathrm{j}$ 's neighbor do not occupied the same channel is given by [15]

$$
\mathrm{P}_{\mathrm{i}, \mathrm{j}}^{\mathrm{a}}=\left(\frac{\mathrm{KP}_{0}-1}{\mathrm{KP}_{0}}\right)^{\left|\mathrm{N}_{\mathrm{i}}\right|}
$$

The probability that the communication from OBU $\mathrm{i}$ to $\mathrm{OBU} \mathrm{j}$ do not collide with primary user is [15]

$$
P_{i, j}^{b}=\frac{P_{0}\left(1-P_{f}\right)}{P_{0}\left(1-P_{f}\right)+\left(1-P_{0}\right) P_{m}}
$$

Hence, the probability of successful communication from $\mathrm{OBU} i$ to $\mathrm{OBU} \mathrm{j}$ is given by [15]

$$
\begin{aligned}
P_{i, j} & =P_{i, j}^{a} P_{i, j}^{b} \\
& =\left(\frac{K P_{0}-1}{K_{0}}\right)^{\left|N_{i}\right|} \frac{P_{0}\left(1-P_{f}\right)}{P_{0}\left(1-P_{f}\right)+\left(1-P_{0}\right) P_{m}}
\end{aligned}
$$


The above equation provides the utility which corresponds to number of packets successfully communicated as discussed in [15].

\section{Proposed Approach: Multiple Antennas Based Modified Approach}

The multiple antennas based modified approach uses multiple antennas at RSUs and OBUs. The antennas provide the resistance to fading which improves the connectivity in terms of increased data rate. The antenna utilization parameter is taken into consideration. Let the utilization parameter for single antenna is 1 . It can be related to channel capacity [16], [17].[17] Proposes the beam forming algorithms for cognitive radio networks for achievable data rate and isequipped with multiple antennas. It is proved that $\mathrm{N}_{\mathrm{t}}^{\mathrm{c}} \times 2\left(\mathrm{~N}_{\mathrm{t}}^{\mathrm{c}}\right.$ transmit antennas at the cognitive transmitter and 2 receive antennas at the cognitive receiver) MIMO link nullifies interference by maximizing data rate. If more than one antenna is used then the value of utilization parameter of multiple antennas is more than one. It depends on the number of antenna used.

Let NA be the number of antennas used in CR VANET at RSUs and OBUs. Then the utilization parameter is given by

$$
U A=\frac{\log \left(\mathrm{N}_{\mathrm{A}}+1\right)}{\log (2)}
$$

If $\mathrm{NA}=1$, then $U A=1 . \mathrm{NA}>1$, then $U A>1$. During $\mathrm{V} 2 \mathrm{R}$ communication, the channel capacity between RSU and $\mathrm{OBU} \mathrm{i}$ is given by

$$
\left(\mathrm{c}_{(\mathrm{i})}\right)_{\mathrm{UA}}=\mathrm{UA}\left[\mathrm{W} \log _{2}\left(1+\beta\left|\mathrm{h}_{\mathrm{i}}\right|^{2}\right)\right]
$$

During V2V communication, the channel capacity is given by

$$
\left(\mathrm{c}_{\mathrm{i}, \mathrm{j}}^{\mathrm{k}}\right)_{\mathrm{UA}}=\mathrm{UA}\left[\hat{\mathrm{W}}_{\mathrm{k}} \log _{2}\left(1+\beta_{\mathrm{i}}\left|\mathrm{h}_{\mathrm{i}, \mathrm{k}}^{\mathrm{k}}\right|^{2}\right)\right], \quad \mathrm{k}=1,2, \ldots \ldots \ldots, \mathrm{K}
$$

The transmission rate using non-cooperative approach is given by

$$
\left(\mathrm{R}_{\mathrm{j}, \mathrm{i}}^{\mathrm{k}}\right)_{\mathrm{UA}}=\mathrm{UA}\left[\left(\frac{\mathrm{T}-\mathrm{K}_{\mathrm{j}} \mathrm{\tau}}{\mathrm{T}}\right) \mathrm{c}_{\mathrm{j}, \mathrm{i}}^{\mathrm{k}}\right] \mathrm{k} \in k_{j}
$$

The OBU selects the channel with largest transmission rate and the channel capacity which is a function of $f\left(k_{j}\right)$.

$$
\text { Let } f\left(k_{j}\right)_{U A}=U A\left[A \ln \left(k_{j}+1\right)\right]
$$

During the coalitional graph game based approach, the probability of successful communication from OBU $\mathrm{i}$ to $\mathrm{OBU} \mathrm{j}$ is given by

$$
\begin{aligned}
{\left[\mathrm{P}_{\mathrm{i}, \mathrm{j}}\right]_{\mathrm{UA}} } & =\mathrm{UA}\left[\left(\mathrm{P}_{\mathrm{i}, \mathrm{j}}^{\mathrm{a}} \mathrm{P}_{\mathrm{i}, \mathrm{j}}^{\mathrm{b}}\right)\right] \\
& =\mathrm{UA}\left[\left(\frac{\mathrm{KP}_{0}-1}{\mathrm{KP}_{0}}\right)^{\left|\mathrm{N}_{\mathrm{i}}\right|} \frac{\mathrm{P}_{\mathrm{o}}\left(1-\mathrm{P}_{\mathrm{f}}\right)}{\mathrm{P}_{\mathrm{o}}\left(1-\mathrm{P}_{\mathrm{f}}\right)+\left(1-\mathrm{P}_{0}\right) \mathrm{P}_{\mathrm{m}}}\right]
\end{aligned}
$$

The above equation provides the utility which corresponds to number of packets successfully communicated as discussed in [15].

\section{NUMERICAL RESULTS}

The performance of various available approaches and proposed approach is simulated and evaluated under various conditions. The main simulation parameters are given in table I.

TABLE I. SIMULATION PARAMETERS

\begin{tabular}{|c|c|}
\hline Definition & Variable \\
\hline Number of antennas used at RSU and OBU & $N_{A}=1 \sim 2$ \\
\hline Number of channels for V2V communication & $\mathrm{K}=1 \sim 10$ \\
\hline Number of sensed channels for V2V communication & $k_{j}=1 \sim \mathrm{k}$ \\
\hline Transmit power of OBUs and RSUs & $\beta=1 \mathrm{~dB}$ \\
\hline Probability of miss detection & $P_{m}=0.1$ \\
\hline Probability of false alarm & $P_{f}=0.1$ \\
\hline Initial distance of OBU before it crosses RSU & $D_{1}=30 \mathrm{~m}$ \\
\hline Distance of OBU after it crosses RSU & $D_{2}=70 \mathrm{~m}$ \\
\hline Bandwidth for V2R channel & $\mathrm{W}=1 \mathrm{unit}$ \\
\hline Bandwidth for V2V channel & $\hat{\mathrm{W}}=1 \mathrm{unit}$ \\
\hline
\end{tabular}




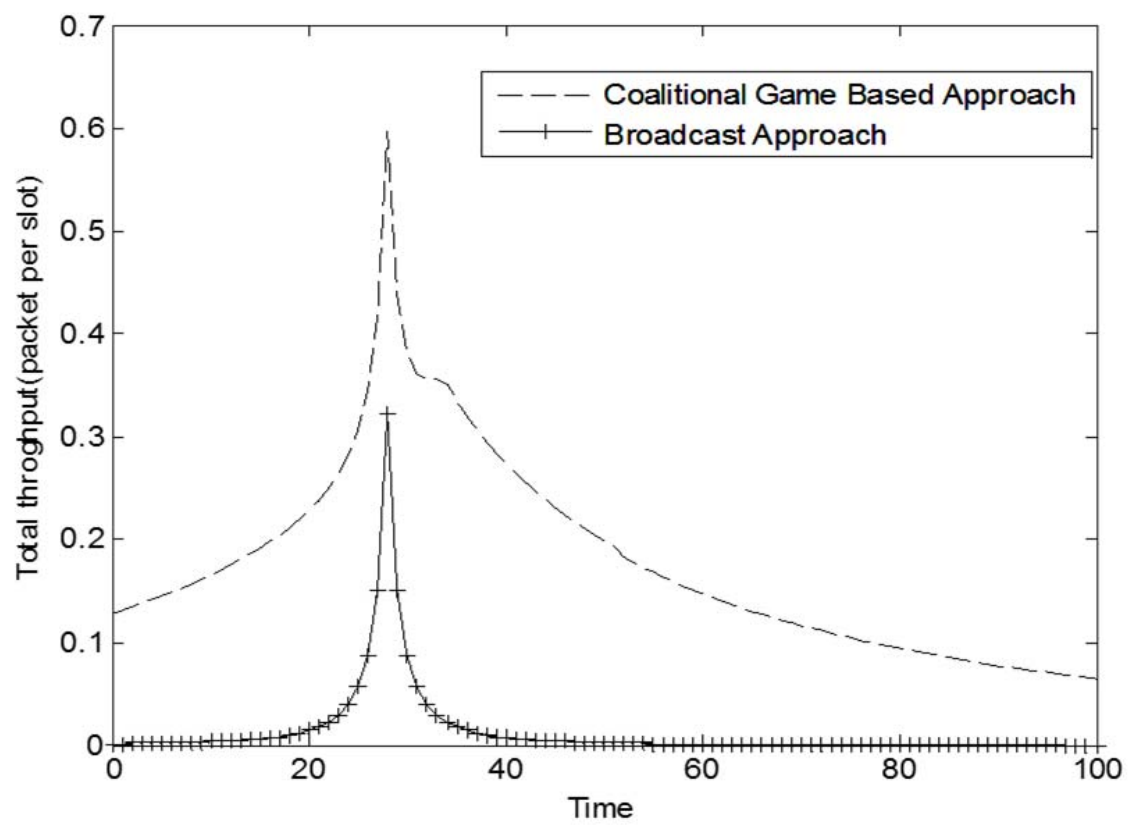

Fig.1. Total throughput as a function of time

Fig.1. shows the total throughput as a function of time for pure broadcast approach and coalitional graph game based approach for CR VANET. It is shown that for pure broadcast scheme, the total throughput increases as OBUs enter in the communication range of RSUs. Its value decreases to zero as OBUs leave this area. The coalitional graph game based approachperforms better in terms of increased throughput. The packets are still transmitted between OBUs even after OBUs leave RSUs area. It is also shown that total throghput increases untill OBUs exchange data and then decreases in both proposed approaches. In brief, coalitional graph game based approach provides better connectivity than pure broadcast approach when packets are handoff in CR VANET.

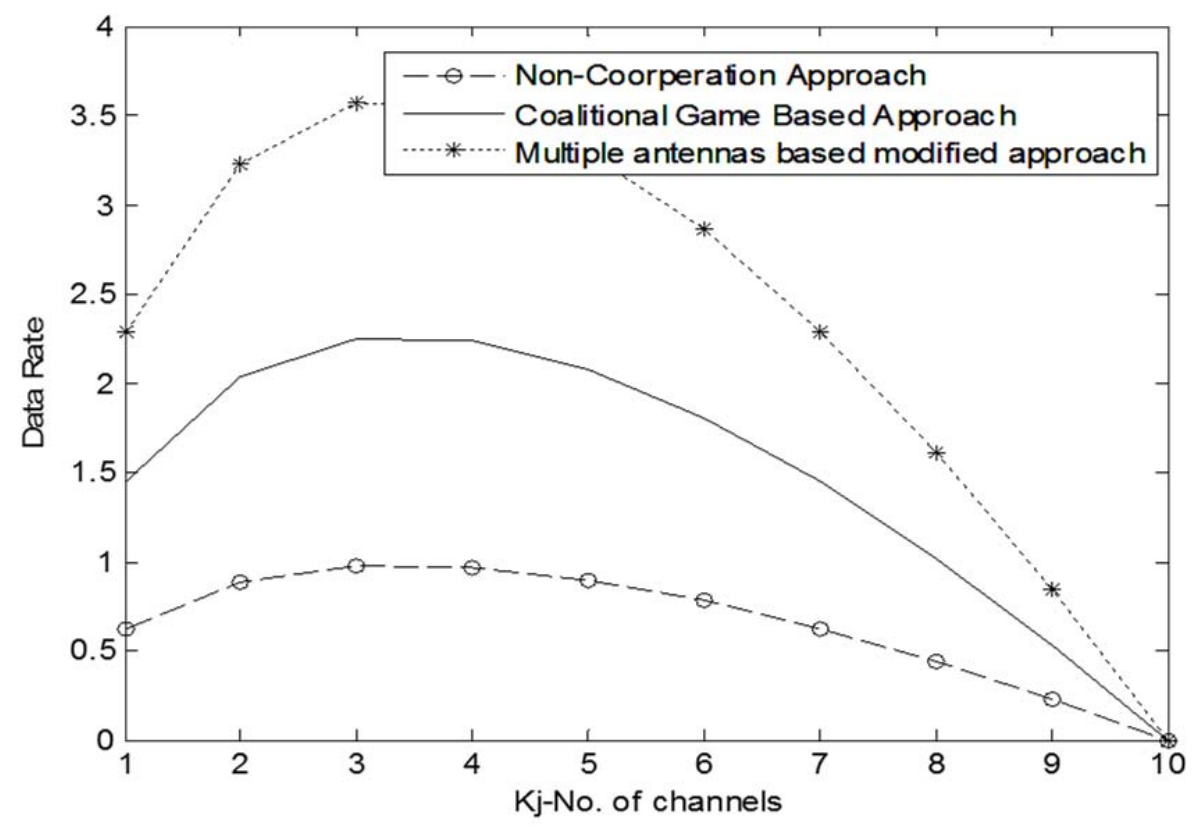

Fig. 2. Data rate as a function of number of sensed channels

Fig. 2 shows that the data rate variation with number of sensed channels. The sensed channels kj varies from 1 to 10 in all the three approaches. As can be seen, the best performance in terms of data rate occurred when $\mathrm{kj}=4$ which coordinates to equation (5) in all the three approaches. Hence, application of multiple antennas based modified approach is correct and applicable to improve the connectivity of CR VANETs. 


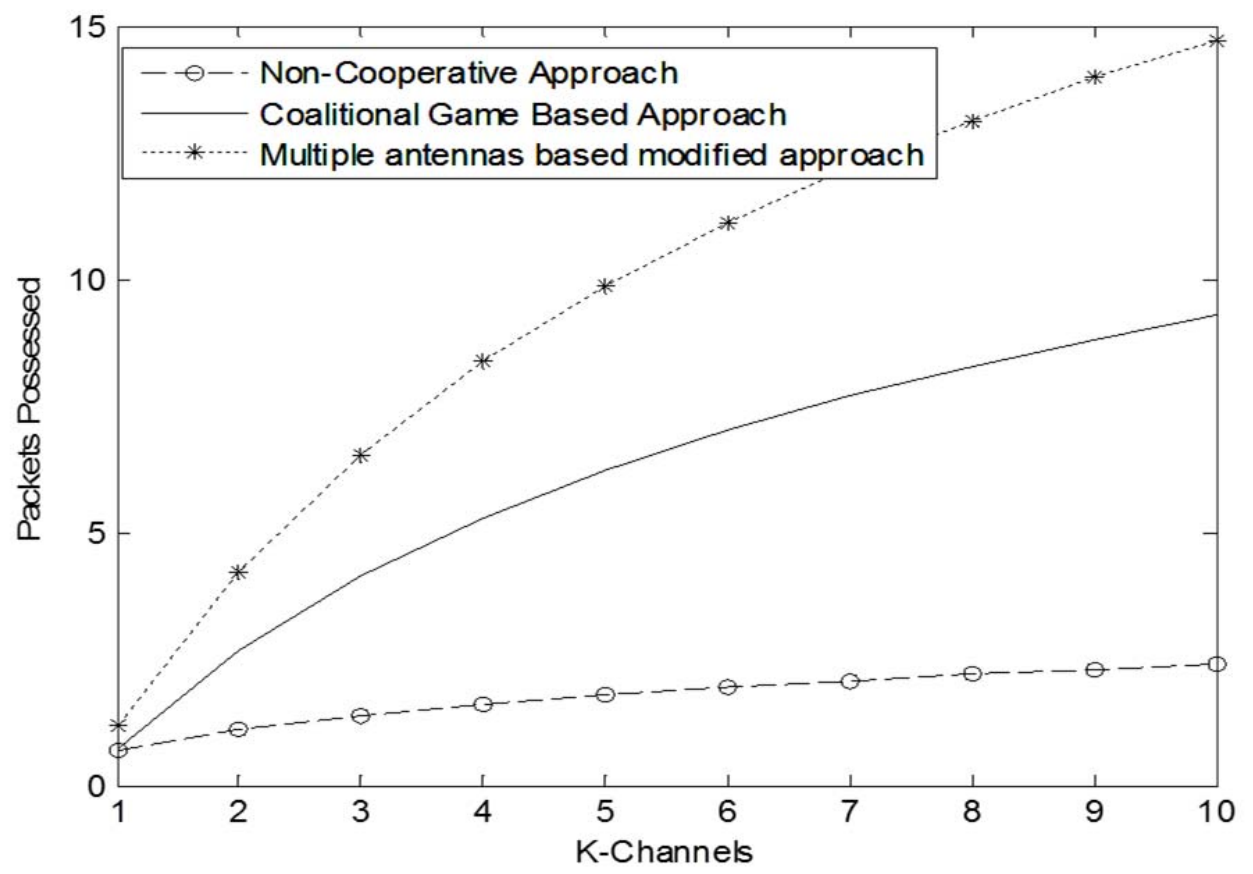

Fig.3. Packet possessed as a function of number of channels

Fig. 3 shows the packets possessed as a function of number of channels. As seen, there is considerable improvement in the connectivity in term of packets possessed with multiple antennas based modified approach compared with other approaches. Its effects are high when the number channels are large. The numbers of channels are large in highway as plenty of holes are there with CR networks. Hence multiple antennas based modified approach is more effective in CR VANETs.

\section{CONCLUSION}

This paper proposes the approach to use of multiple antennas to improve the connectivity of CR VANETs during handoffs. It shows that coalitional graph game based approach provides better connectivity than pure broadcast approach when packets are handoffs in CR VANETs. The packets are still transmitted between OBUs even after OBUs leave RSUs area using V2V communication. But major disadvantage with coalitional graph game based approach is the sharing of information among nodes because of its cooperative nature. Hence the modification is done in available non-cooperative approach and coalitional graph game based approach by introducing antenna utilization parameter which uses two number of antenna at OBUs and RSUs. It is found that this approach works correctly and finds considerable improvement in the data rates and packets possessed. Thus itreduces the interference and improving the connectivity in CR VANETs.In future work, itcan be interesting to add the amount of cooperation in the above work. This can be done by adding the concept of percolation for connectivity in cooperative secondary networks. The values of percolation threshold decide the amount of cooperation. The secondary network may have other secondary network acting as a relay. The less power is required to sustain the same level of connectivity using cooperation or percolation.

\section{REFERENCES}

[1] C.-J. Kim, J. Kim and C. Pyo, "Dynamic Spectrum Access/Cognitive Radio Activities in Korea," in Proc. IEEE New Frontiers in Dynamic SpectrumSymp.,April 2010, pp. 1-5.

[2] H. Bezabih, B. Ellingsæter, J. Noll and T. Maseng, "Digital broadcasting: Increasing the white space spectrum using TV receiver information," IEEE Veh.Technol Mag, vol. 7, pp. 24-30, March 2012.

[3] B. Wang and K. J. Ray Liu, "Advances in Cognitive Radio Networks: A Survey," IEEE J. Sel. Topics Signal Process., vol. 5, no. 1, pp.5-23, February 2011.

[4] Z. M. Liu, N. Nasser and H. S. Hassanein, "Intelligent spectrum assignment and migration in cognitive radio network," EURASIP J. Wireless Communication andNetwork., vol. 2013, no. 200, 2013.Available:http://jwcn.eurasipjournals.com/content/2013/1/200.

[5] W. Jouini, C. Moy and J. Palicot, "Decision making for cognitive radio equipment: analysis of the first 10 years of exploration," EURASIP J. Wireless Communication andNetwork, vol. 2012, no. 26, 2012. Available: http://jwcn.eurasipjournals.com/content/2012/1/26.

[6] C. R. Stevenson, G. Chouinard, Z. Lei, W. Hu, S. J. Shellhammer and W. Caldwell, "IEEE 802.22: The first cognitive radio wireless regional area network standard," IEEE Communication. Mag.,vol. 47, no. 1, pp 130-138, April 2009.

[7] J. Mitola and G. Q. Maguire, "Cognitive radio: Making software radios more personal," IEEE Pers.Communication., vol. 6, no. 4, pp. 13-18, August, 1999.

[8] I. F. Akyildiz, W.-Y Lee, M. C. Vuran and S. Mohanty, "A Survey on spectrum management in cognitive radio networks,"IEEE Communication. Mag., vol. 46, no.4, pp. 40-48, April 2008. 
[9] A. Prakash and R.Tripathi, "Vehicular Ad Hoc Networks toward Intelligent Transport Systems," in Proc.IEEE TENCON-2008, Hyderabad, India, Nov. 2008, pp.1-6.

[10] A. Prakash, et. al. "Vehicle assisted cross-layer handover scheme in NEMO-based VANETs (VANEMO)," Int. J. Internet Protocol Technol., vol. 6, nos. 1/2, pp.83-95, 2011.

[11] A. Prakash, R. Verma, R. Tripathi,and K.Naik,“A seamless handover scheme for vehicles across heterogeneous networks,” Int. J. Communication andNetwork .andDistribution.Syst., vol. 8, nos. 1/2, pp.4-23, 2012.

[12] W. C.Ao, S.-M.Cheng and K.-C. Chen, "Connectivity of Multiple Cooperative Cognitive Radio Ad Hoc Networks," IEEE J. Sel. Areas Communication., vol. 30, no. 2, pp. 263-270, Feb. 2012.

[13] K.D.Singh, P. Rawat and J.-M.Bonnin, "Cognitive radio for vehicular ad hoc networks (CR-VANETs): approaches and challenges,"EURASIP J. Wireless Communication andNetwork., vol 2014, no. 49, 2014 . Available: http://jwcn.eurasipjournals.com/content/2014/1/49.

[14] V. Srinivasan, P. Nuggehalli, C.-F.Chiasserini and R. R. Rao, "An Analytical Approach to the Study of Cooperation in Wireless Ad Hoc Networks,”IEEE Trans. Wireless Communication.,vol. 4, no. 2, pp. 722-733, 2005.

[15] T. Wang, L. Song and Z. Han, "Coalitional Graph Games for Popular Content Distribution in Cognitive Radio VANETs," IEEETransaction. Veh.Technol., vol. 62, no. 8, pp.4010-4019, Oct. 2013.

[16] S. Loyka and A. Kouki, "The Impact of Correlation on Multi-Antenna System Performance: Correlation Matrix Approach," in Proc. IEEE VTC 2001, Atlantic City, NJ, vol. 2, 2001, pp. 533-537.

[17] S. Yiu, C.-B.Chae, K. Yang and D. Calin, "Uncoordinated Beamforming for Cognitive Networks," IEEE Trans. Communication., vol. 60, no. 5, pp.1390-1397, May 2012.

\section{Author Profile}

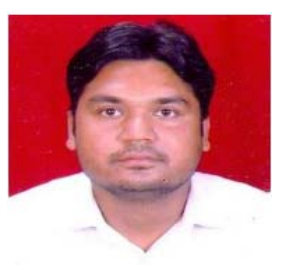

Krishan Kumar received his $\mathrm{PhD}$. degree from Department of Electronics and Communication Engineering, Motilal Nehru National Institute of Technology, Allahabad, India under Quality Improvement Programme (QIP) scheme supported by All India Council for Technical Education (AICTE) New Delhi, Government of India. $\mathrm{He}$ has received M.Tech. degree in Electronics and Communication Engineering, National Institute of Technology, Kurukshetra, India in 2005. Currently, is working as an Assistant Professor, National Institute of Technology, Hamirpur, India since 2006. He has number of publications in SCI and Scopus indexed journals. His research interests are in the area of wireless and mobile networks: mobility management aspects and cognitive radio networks.

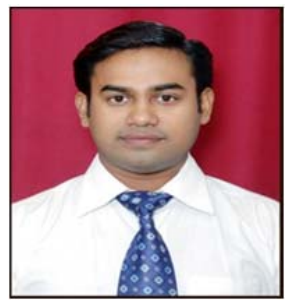

Mani Shekhar is currently pursuing his PhD. Degree from Electronics and Communication Engineering Department, National Institute of Technology, Hamirpur, India. He has obtained his M Tech. degree in Communication systems and networks from National Institute of Technology Hamirpur, in 2011. His research is concern the Conginitive radio, Vertical Handover and Secure Mobility Management for Next Generation Networks. 